\title{
BMJ Global Health Analysis of out-of-pocket costs associated with hospitalised injuries in Vietnam
}

\author{
Ha Nguyen, ${ }^{1}$ Rebecca Ivers, ${ }^{2}$ Stephen Jan, ${ }^{2}$ Cuong Pham ${ }^{3}$
}

To cite: Nguyen $\mathrm{H}$, Ivers $\mathrm{R}$, Jan $\mathrm{S}$, et al. Analysis of outof-pocket costs associated with hospitalised injuries in Vietnam. BMJ Global Health 2017;2: e000082. doi:10.1136/ bmjgh-2016-000082

Received 5 May 2016 Revised 5 November 2016 Accepted 18 December 2016

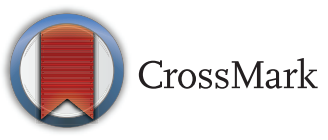

${ }^{1}$ School of Health Sciences, University of South Australia, Adelaide, South Australia, Australia

${ }^{2}$ The George Institute for Global Health, University of Sydney, Sydney, New South Wales, Australia

${ }^{3}$ The Centre for Injury Policy and Prevention Research, Hanoi School of Public Health, Hanoi, Vietnam

Correspondence to

Dr Ha Nguyen;

ha.nguyen2@unisa.edu.au

\section{ABSTRACT}

Background: In Vietnam, over 39000 people die and millions are hospitalised due to injuries every year. Injuries create a significant financial burden for families. In this study, we estimated out-of-pocket payment for medical care of injuries and the role of health insurance in containing such costs.

Method: A prospective cohort study of 892 hospitalised injury patients admitted to a provincial general hospital in 2010 in Vietnam was conducted. Data on demographic, injury characteristics and costs by specific categories paid out-of-pocket by patients were included in the analyses. Generalised linear models with log link and $\gamma$ distribution were employed to examine the associations between insurance status and total costs.

Results: The average total medical care costs paid out-of-pocket by patients during hospitalisation were over US\$270. Major drivers of the costs related to surgery (nearly $25 \%$ ), diagnostic test/examination $(24 \%)$ and drugs $(23 \%)$. Burns incurred the highest total costs during hospitalisation (\$321) and assault incurred the lowest (\$167). Total costs were higher for more severe injuries and those that required a more complex surgery. Patients using health insurance paid less those who did not. However, there were no statistically significant associations between health insurance and total costs, costs for surgery, diagnostic tests/examinations or drugs.

Conclusions: The study provides estimates and distribution of medical care costs for injuries in hospital. In addition to ongoing efforts in prevention, the study provides further evidence on the limited benefits of health insurance in protecting patients and their family from the high costs of hospitalisation in Vietnam.

\section{INTRODUCTION}

Injury is one of the most challenging public health issues in the 21st century. Over 14000 people die because of injuries every day or about 5 million people every year. Injuries account for $\sim 9 \%$ of the world's mortality and 1.7 times the number of deaths resulting from malaria, tuberculosis and HIV/AIDS combined. ${ }^{1}$ Road traffic injuries (RTIs)

\section{Key questions}

What is already known about this topic?

- Injury is one of the major causes of burden of disease and mortality in low and middle-income countries (LMICs).

- In LMICs, large proportion of medical care costs is paid out-of-pocket by patients.

- Costs for injury treatment can result in catastrophic expenditure in many families in LMICs.

What are the new findings?

- Major cost categories, paid out-of-pocket by injury patients, were costs for surgery, diagnostic tests/examinations and drugs.

- There was no statistically significant effect of health insurance in reducing the costs for surgery, diagnostic tests/examinations and drugs for injury treatment.

Recommendations for policy

- Measures to prevent injuries need to be re-enforced.

- A system of regulation and supervision to avoid the issue of provider-induced supply of unnecessary care during medical treatment need to be in place and properly enforced.

- Barriers in the usage of health insurance must be investigated and addressed.

ranked ninth among the leading causes of death in 2015, and are predicted to rise to the seventh by $2030 .^{2}$ One of the most serious concerns of injuries is that more than $90 \%$ of fatal and majority of non-fatal injuries occurs in low and middle-income countries (LMICs), which also have limited capacity in prevention and treatment. ${ }^{1}$

Similar to other LMICs, Vietnam bears a significant burden of injuries. Annually, there are over 39000 injury deaths, accounting for over $10 \%$ of all deaths. ${ }^{3}$ Among 10 leading causes of death, 3 are injury-related, including RTIs, drowning and suicide. Non-fatal injuries are also significant. A recent national injury survey estimated that in 2010, nearly 1.8 million non-fatal injuries 
( $2 \%$ of population) required medical attention and/or at least 1 day off from work or school. Among these, $36 \%$ or 648000 cases were hospitalised for at least 1 day. ${ }^{4}$ Injuries also create economic burden to the victims and their families. In a study on injury costs, it was found that $26 \%$ of the study sample faced catastrophic expenditure because of their injuries, ${ }^{5}$ which is over 2.5 times the average level in Vietnam $(10.5 \%){ }^{6}$

The economic burden of injuries will become larger in Vietnam because of the increasing costs of healthcare services. Since the introduction of user fee in 1989, people were required to begin paying fee for healthcare service. ${ }^{7}$ To improve access to healthcare and to mitigate the negative impact of user fee, national health insurance was introduced in 1992. On average, the insurance covers about $85 \%$ of the total service cost. The coverage has increased steadily over time, from 5\% in 1993 to $60 \%$ in $2010 . .^{78}$

The current study aims to determine the distribution of the direct medical costs, including those for surgery, emergency department, test and examinations, drug, equipment, transportation and the hospital stay. By evaluating where the resources are being used and calculating the actual costs of these services, the major contributors to the hospitalisation costs can be identified. We then examine the role of health insurance on individual major cost contributors which then can be used as evidence on the effect of health insurance in protecting injury patients from increased treatment costs.

\section{METHOD}

\section{Study design and participants}

A prospective cohort study was conducted with injury patients recruited consecutively from Thai Binh General Hospital between 1 January 2010 and 31 August 2010 and followed for 1 year after discharge. Thai Binh General Hospital is the largest trauma provincial hospital with 440 beds in Thai Binh province, located $\sim 100 \mathrm{~km}$ south of Hanoi, the capital city of Vietnam. As the largest trauma hospital, Thai Binh General Hospital receives the majority of injury patients requiring inpatient hospitalisation. Estimating from the data from the Provincial Department of Health, during the study recruitment period (January to August 2010), there were more than 1120 adult injuries requiring inpatient hospitalisation for at least 1 day. Among these, 982 individuals $(88 \%)$ were admitted (directly and indirectly) into Thai Binh General Hospital. ${ }^{9}$ A more detailed description of the study design, participants and data collection of the project has been previously reported. ${ }^{10}$ In reporting mean costs across all patients recruited and by covering two-third the time of the year, it was expected that the effect of not including patients in the other one-third of the year would be minimal.

Participants recruited were patients admitted to Thai Binh General Hospital as a result of an injury and hospitalised for at least 1 day. Participants were aged 18 years or older and were residing in the province at the time of recruitment to improve the feasibility of follow-up. During the recruitment period, 918 participants who met the selection criteria were approached and 892 $(97 \%)$ consented to participate into the study.

The ethics application for the study was reviewed and approved by the Human Ethics Committee in University of Sydney, Australia, the Hanoi School of Public Health Ethics Committee and Thai Binh General Hospital in Vietnam.

\section{Data}

In this paper, we report and analyse out-of-pocket costs incurred during participants' hospital stay. The following cost categories were collected: surgery, emergency department, test (including laboratory tests and radiology/pathology examinations), drugs, equipment, transportation and hospital stay (ie, patient's accommodation). While most of these were collected from actual hospital bill, additional costs for drugs, equipment and transportation were also collected from interview with patients and their caretakers because during hospitalisation, patients could also get these outside of hospital (eg, over-the-counter drugs, self-transportation to hospital).

Other data collected include age, gender, occupation, insurance status, injury characteristics and diagnosis (including external cause of injury, principal body position injured, injury severity measured by the maximum abbreviated injury scale (MAIS)). Participants were categorised into groups, including 'non-surgical' if no surgery was performed; or 'minor surgical' if a surgical procedure was performed and only skin and/or connective tissue was resected; or 'major surgical' if a more extensive resection was performed such as an open reduction or internal fixation of broken bone.

\section{Statistical analysis}

Costs for individual patient were determined and summed by category. We then calculated the mean and percentage of each cost category by patient demographics and injury characteristics and diagnosis. In examining the effect of insurance status on total hospitalisation costs and major cost contributors, we examined the ordinary least squares (OLS) of the log transformation and the generalised linear models (GLM) with log links to account for the empirical non-negativity and positively skewed distribution of costing data. ${ }^{11}$ Heteroskedasticity of residuals was checked by White's test and the Breusch-Pagan test. ${ }^{12}$ Heteroskedasticity can result in bias and inefficiency in OLS models. Because of the existence of heteroskedasticity, GLM models with $\log$ link and $\gamma$ distribution were selected to examine factors associated with increased cost. The $\gamma$ distribution was verified by the subsequent modified Park tests. ${ }^{11}$ We examined unadjusted models with insurance status as the only independent variable and adjusted models with insurance status as independent variable controlling for 
demographic and injury characteristics. Only characteristics with significant association $(\mathrm{p}<0.05)$ in univariable models and identified to be associated with costs in prior studies were included in adjusted models. The significance level was set at 0.05 . Residual deviance was reported as a measure of goodness of fit of a GLM, testing the fitted model against the saturated model. ${ }^{13}$ Or rather, it is a measure of badness of fit, thus a lower value indicates a better fit. STATA 12 (Stata Corporation, College Station, Texas, USA) was employed to perform all analyses.

\section{RESULTS}

Table 1 summarises the demographic characteristics of participants in the study. The majority of participants was in the group aged $18-29$ years $(27.8 \%)$, followed by participants the 60 year and over group. Males were dominant in this study, accounting for over $70 \%$. Regarding the distribution by occupation categories, farmers comprised the largest group with 464 individuals or over $50 \%$. White-collar workers or those working in office contributed only $8 \%$. In terms of health insurance, $25 \%$ of participants had and used it during their hospital stay.

The distribution of the sample by injury characteristics is presented in table 2. RTIs and falls accounted for the largest proportions $53 \%$ and $29 \%$, respectively. The rest (18\%) included burns (2\%), sharp object (4\%), blunt object (4\%), assault (5\%) and others (including animal bite and electrocution) (3\%). Injuries with the MAIS of 5 (those in critical or uncertain survival condition)

Table 1 Demographic characteristics of participants

\begin{tabular}{lc}
\hline & $\mathbf{n}(\%)$ \\
\hline Total & $892(100)$ \\
\hline Age & \\
\hline $18-29$ & $248(27.8)$ \\
$30-39$ & $134(15.0)$ \\
$40-49$ & $162(18.2)$ \\
\hline $50-59$ & $133(14.9)$ \\
\hline $60+$ & $215(24.1)$ \\
\hline Gender & \\
\hline Male & $634(71.1)$ \\
\hline Female & $258(28.9)$ \\
\hline Occupation & \\
\hline White-collar worker & $74(8.3)$ \\
\hline Blue-collar worker & $139(15.6)$ \\
\hline Farmer & $464(52)$ \\
\hline Retiree/unemployed & $215(24.1)$ \\
\hline Insurance & \\
\hline Yes & $224(25.1)$ \\
\hline No & $668(74.9)$ \\
\hline
\end{tabular}

accounted for nearly $3 \%$, while $40.1 \%$ had sustained injuries with MAIS of 2 (moderate) and another $41.4 \%$ with MAIS of 3 (serious). Injuries with MAIS of 1 (minor) and MAIS of 4 (life threatening) contributed $<10 \%$ each. Regarding the principal body region injured (or the most severely injured region), the most common was the head $(34 \%)$, followed by the lower extremities $(28 \%)$ and the upper extremities (17\%). Minor surgery was performed in $39.7 \%$ of participants, while $31.4 \%$ had undergone major surgery. In terms of admission route, $55.2 \%$ of participants admitted directly, $37.8 \%$ from lower level hospitals and $7.1 \%$ from higher level hospitals.

Table 3 shows the total medical care costs paid out-of-pocket by participants at discharge and the distribution of these costs by seven major categories stratified by participant demographic characteristics. Total costs generally increased with age with the highest costs for participants aged 60 years and older (US\$ 314.1). Females incurred costs which were $\sim 20 \%$ higher than those incurred by males. White-collar workers were the group with highest total costs (US\$ 283.8), while bluecollar workers were the group with lowest total costs (US \$235.6). Regarding the insurance usage status, the total paid was lower for participants used their insurance. It was commonly $<90 \%$ of that paid by those who did not use their insurance.

Across seven cost categories, it can be seen that the three major drivers were surgery cost, accounted for the largest proportion of the total $(24.8 \%)$, cost for diagnostic tests or examinations (23.9\%) and that for drugs $(23.4 \%)$. Categories with the lowest proportions in the total costs were costs incurred in the emergency department and for patient transport, representing $5.1 \%$ and $5.4 \%$, respectively. Despite variations across the distribution of each cost category by demographic characteristics, surgery, tests/examinations and drugs were the main cost drivers regardless of participants' age, gender or occupation. Participants with health insurance appeared to pay lower amounts for almost all cost categories than those without health insurance. The only category that without health insurance paid less was the cost for hospital days.

Costs by injury characteristics and their distribution across cost categories are summarised in table 4. In terms of external cause, burn was the injury incurred the highest costs, US\$321, which was nearly twice the costs incurred by assault, the least costly injury. Drugs were the major cost driver for burns (US\$ 91.0 or over $28 \%$ of the total costs, the highest proportions compared with all other external cause). Surgery was highest among fall-related injuries $(\$ 74.5)$. However, this was represented only $25 \%$ of the total costs. Surgery accounted for the largest proportion $(30.6 \%)$ of the total costs in sharp object-related injuries. Diagnostic test and examination was largest in fall injuries, in terms of absolute value and proportion of the total (US\$ 73.6 and $24.9 \%$ ). 
Table 2 Injury characteristics

\begin{tabular}{|c|c|c|c|c|c|c|c|c|}
\hline Total & $\begin{array}{l}\text { RTI } \\
\text { (V01-V89) } \\
\text { n (\%) } \\
477\end{array}$ & $\begin{array}{l}\text { Fall } \\
\text { (W00-W19) } \\
\text { n (\%) } \\
261\end{array}$ & $\begin{array}{l}\text { Burn } \\
\text { (X00-X19) } \\
\text { n (\%) } \\
22\end{array}$ & $\begin{array}{l}\text { Sharp } \\
\text { object } \\
\text { (W25-W27) } \\
\text { n (\%) } \\
32\end{array}$ & $\begin{array}{l}\text { Blunt object } \\
\text { (W20-W22) } \\
\text { n (\%) } \\
33\end{array}$ & $\begin{array}{l}\text { Assault } \\
\text { (X85-Y09) } \\
\text { n (\%) } \\
43\end{array}$ & $\begin{array}{l}\text { Other } \\
\text { injuries } \\
\text { n (\%) } \\
24\end{array}$ & $\begin{array}{l}\text { All } \\
\text { injuries } \\
\text { n (\%) } \\
892\end{array}$ \\
\hline \multicolumn{9}{|l|}{ MAIS } \\
\hline 1 & $33(6.9)$ & $15(5.7)$ & $4(18.2)$ & $2(6.3)$ & $6(18.2)$ & 9 (20.9) & $6(25)$ & $75(8.4)$ \\
\hline 2 & $203(42.6)$ & $96(36.8)$ & $11(50.0)$ & $16(50.0)$ & $9(27.3)$ & $14(32.6)$ & $9(37.5)$ & $358(40.1)$ \\
\hline 3 & $184(38.6)$ & $126(48.3)$ & $6(27.3)$ & $13(40.6)$ & $14(42.4)$ & $17(39.5)$ & $9(37.5)$ & $369(41.4)$ \\
\hline 4 & $38(8.0)$ & $19(7.3)$ & $1(4.5)$ & $1(3.1)$ & $4(12.1)$ & $1(2.3)$ & $0(0.0)$ & $64(7.2)$ \\
\hline 5 & $19(4.0)$ & $5(1.9)$ & $0(0.0)$ & $0(0.0)$ & $0(0.0)$ & $2(4.7)$ & $0(0.0)$ & $26(2.9)$ \\
\hline \multicolumn{9}{|l|}{ Principal injured region } \\
\hline Head & $207(43.4)$ & $73(28)$ & $0(0.0)$ & $2(6.3)$ & $9(27.3)$ & $10(23.3)$ & $2(8.3)$ & $303(34)$ \\
\hline Face & $50(10.5)$ & $5(1.9)$ & $6(27.3)$ & $2(6.3)$ & $4(12.1)$ & $13(30.2)$ & $2(8.3)$ & $82(9.2)$ \\
\hline Spine & $12(2.5)$ & $26(10.0)$ & $0(0.0)$ & $0(0.0)$ & $3(9.1)$ & $0(0.0)$ & $0(0.0)$ & $41(4.6)$ \\
\hline Thorax/abdomen & $35(7.3)$ & $17(6.5)$ & $3(13.6)$ & $0(0.0)$ & $1(3.0)$ & $2(4.7)$ & $3(12.5)$ & $61(6.8)$ \\
\hline Upper extremity & $58(12.2)$ & $24(9.2)$ & $6(27.3)$ & $23(71.9)$ & $11(33.3)$ & $17(39.5)$ & $15(62.5)$ & $154(17.3)$ \\
\hline Hip/lower extremity & $115(24.1)$ & $116(44.4)$ & $7(31.8)$ & $5(15.6)$ & $5(15.2)$ & $1(2.3)$ & $2(8.3)$ & $251(28.1)$ \\
\hline \multicolumn{9}{|l|}{ Surgery } \\
\hline Non-surgery & $147(30.8)$ & $82(31.4)$ & $9(40.9)$ & $3(9.4)$ & $4(12.1)$ & $6(14.0)$ & 7 (29.2) & $258(28.9)$ \\
\hline Minor surgery & $204(42.8)$ & $83(31.8)$ & $12(54.5)$ & $12(37.5)$ & $17(51.5)$ & $20(46.5)$ & $6(25.0)$ & 354 (39.7) \\
\hline Major surgery & $126(26.4)$ & $96(36.8)$ & $1(4.5)$ & $17(53.1)$ & $12(36.4)$ & $17(39.5)$ & $11(45.8)$ & $280(31.4)$ \\
\hline \multicolumn{9}{|l|}{ Admission route } \\
\hline Direct & $272(57.0)$ & $133(51.0)$ & $12(54.5)$ & $21(65.6)$ & $20(60.6)$ & $21(48.8)$ & $13(54.2)$ & $492(55.2)$ \\
\hline Indirect-lower & $160(33.5)$ & $112(42.9)$ & $10(45.5)$ & $11(34.4)$ & $12(36.4)$ & $21(48.8)$ & $11(45.8)$ & 337 (37.8) \\
\hline Indirect-higher & $45(9.4)$ & $16(6.1)$ & $0(0.0)$ & $0(0.0)$ & $1(3.0)$ & $1(2.3)$ & $0(0.0)$ & $63(7.1)$ \\
\hline
\end{tabular}

Total costs increased with the MAIS score or the severity of injury. The trend was also consistent across all cost categories. The total costs were highest for participants with principal body region injured being the head, the upper and the lower extremities. These were also the regions incurred the highest costs for surgery, tests and examinations, and drugs. It was obvious that participants who just required superficial treatment of their injuries incurred no costs for surgery. Those who required major surgery incurred total costs significantly higher than those who did not have any surgery or just had minor surgery. It can be seen that for all categories, costs for those with major surgery were always higher than costs for those with minor or no surgery. Regarding transfer status, patients who admitted directly to the hospital appears to have lower costs than those transferred from other hospital, either from lower or higher level hospitals. The trend of lower costs among directly admitted patient was also consistent across all cost categories.

Analyses on the effect of health insurance on costs paid out-of-pocket during hospitalisation (total and three major cost drivers) are shown in table 5. The exponentiation of the regression coefficient $(\exp (b))$ in the table represents the relative difference between the costs paid by participants with and without insurance. A value of $<1$ indicates that the costs paid by participants with insurance were less than the costs paid by those without insurance. It can be seen that, for all cost categories, participants with insurance appear to pay lower out-of-pocket cost than those without insurance. No model, including unadjusted and adjusted for total costs, and three major cost categories, had statistically significant associations between insurance and reduced costs. In other words, with the same demographic and injury characteristics, costs paid out-of-pocket by patients with insurance were not statistically significantly different from those paid by patients without insurance. This was the same for total, surgery, diagnostic test/examination and drug costs. The relative goodness of fit of the unadjusted and adjusted models was shown by the residual deviance. At lower values, the adjusted models were generally better fit than the unadjusted ones.

\section{DISCUSSION}

The current study provides detailed analyses on the distribution of medical care costs, total and major cost categories, paid out-of-pocket by injury patients during hospitalisation in a provincial hospital in Vietnam. We found that the major drivers of medical care costs 
Table 3 Out-of-pocket costs (amount in \$US and percentage) by demographic characteristics

\begin{tabular}{|c|c|c|c|c|c|c|c|c|}
\hline Total & $\begin{array}{l}\text { Total medical } \\
\text { care } \\
270.6(100 \%)\end{array}$ & $\begin{array}{l}\text { Surgery } \\
67.1(24.8 \%)\end{array}$ & $\begin{array}{l}\text { Tests/ } \\
\text { examinations } \\
64.7(23.9 \%)\end{array}$ & $\begin{array}{l}\text { Drugs } \\
63.2(23.4 \%)\end{array}$ & $\begin{array}{l}\text { Equipment } \\
21.4(7.9 \%)\end{array}$ & $\begin{array}{l}\text { Emergency } \\
\text { department } \\
13.8(5.1 \%)\end{array}$ & $\begin{array}{l}\text { Transport } \\
14.5(5.4 \%)\end{array}$ & $\begin{array}{l}\text { Hospital } \\
\text { days } \\
24.3(9 \%)\end{array}$ \\
\hline \multicolumn{9}{|l|}{ Age } \\
\hline $18-29$ & $248.1(100 \%)$ & $64.2(25.9 \%)$ & $58.3(23.5 \%)$ & $55.6(22.4 \%)$ & $21.3(8.6 \%)$ & $13.5(5.5 \%)$ & $13(5.2 \%)$ & $22.2(8.9 \%)$ \\
\hline 30-39 & $269(100 \%)$ & $61.9(23 \%)$ & $61(22.7 \%)$ & $59.2(22 \%)$ & $19.9(7.4 \%)$ & $22.7(8.4 \%)$ & $13.8(5.1 \%)$ & $22.3(8.3 \%)$ \\
\hline $40-49$ & $263.4(100 \%)$ & $65.1(24.7 \%)$ & $59(22.4 \%)$ & $66.4(25.2 \%)$ & $21.1(8 \%)$ & $10.4(4 \%)$ & $15.7(6 \%)$ & $25.8(9.8 \%)$ \\
\hline $50-59$ & $252.5(100 \%)$ & $59.1(23.4 \%)$ & $61.1(24.2 \%)$ & $65(25.7 \%)$ & $18.1(7.2 \%)$ & $10.7(4.2 \%)$ & $12(4.8 \%)$ & $23.7(9.4 \%)$ \\
\hline $60+$ & $314.1(100 \%)$ & $80.1(25.5 \%)$ & $81.1(25.8 \%)$ & $70.8(22.5 \%)$ & $24.6(7.8 \%)$ & $13.2(4.2 \%)$ & $17.2(5.5 \%)$ & $27.1(8.6 \%)$ \\
\hline \multicolumn{9}{|l|}{ Gender } \\
\hline Male & $256(100 \%)$ & $60.4(23.6 \%)$ & $61.5(24 \%)$ & $59.7(23.3 \%)$ & $20.4(8 \%)$ & $14.2(5.5 \%)$ & $14.2(5.6 \%)$ & $23.4(9.1 \%)$ \\
\hline Female & $306.4(100 \%)$ & $83.5(27.2 \%)$ & $72.7(23.7 \%)$ & $71.8(23.4 \%)$ & $23.8(7.8 \%)$ & $13(4.2 \%)$ & $15.1(4.9 \%)$ & $26.6(8.7 \%)$ \\
\hline \multicolumn{9}{|l|}{ Occupation } \\
\hline White-collar worker & $299.6(100 \%)$ & $71.4(23.8 \%)$ & $64.8(21.6 \%)$ & $79.1(26.4 \%)$ & $20.2(6.7 \%)$ & $12.8(4.3 \%)$ & $11.4(3.8 \%)$ & $25.5(8.5 \%)$ \\
\hline Blue-collar worker & $235.6(100 \%)$ & $61.9(26.3 \%)$ & $53.1(22.5 \%)$ & $50(21.2 \%)$ & $19.8(8.4 \%)$ & $15.1(6.4 \%)$ & $13.4(5.7 \%)$ & $22.3(9.5 \%)$ \\
\hline Farmer & $270.3(100 \%)$ & $66.6(24.6 \%)$ & $63.5(23.5 \%)$ & $65.9(24.4 \%)$ & $20.9(7.7 \%)$ & $14.3(5.3 \%)$ & $14.6(5.4 \%)$ & $23.9(8.8 \%)$ \\
\hline $\begin{array}{l}\text { Retiree/ } \\
\text { unemployed }\end{array}$ & $283.8(100 \%)$ & $70.1(24.7 \%)$ & 75 (26.4\%) & $60.4(21.3 \%)$ & 23.9 (8.4\%) & 12.5 (4.4\%) & $15.9(5.6 \%)$ & 26 (9.2\%) \\
\hline \multicolumn{9}{|l|}{ Insurance } \\
\hline No & 279.3 (100\%) & $70.2(25.1 \%)$ & 66 (23.6\%) & $65.2(23.3 \%)$ & $22.2(7.9 \%)$ & $14.2(5.1 \%)$ & $15.6(5.6 \%)$ & $23.9(8.5 \%)$ \\
\hline Yes & $244.5(100 \%)$ & $57.7(23.6 \%)$ & $61.1(25 \%)$ & $57.3(23.5 \%)$ & 19 (7.8\%) & $12.7(5.2 \%)$ & $11.1(4.6 \%)$ & $25.5(10.4 \%)$ \\
\hline
\end{tabular}




\begin{tabular}{|c|c|c|c|c|c|c|c|c|}
\hline & Total medical care & Surgery & Tests/examinations & Drugs & Equipment & Emergency department & Transport & Hospital days \\
\hline \multicolumn{9}{|l|}{ External cause } \\
\hline RTI & $270.2(100 \%)$ & $65.4(24.2 \%)$ & $64.6(23.9 \%)$ & $59.8(22.1 \%)$ & $21.9(8.1 \%)$ & $16.2(6.0 \%)$ & $16.0(5.9 \%)$ & $24.1(8.9 \%)$ \\
\hline Fall & $297.7(100 \%)$ & $74.5(25.0 \%)$ & $73.6(24.7 \%)$ & $75.2(25.3 \%)$ & $22.4(7.5 \%)$ & $12.2(4.1 \%)$ & $14.0(4.7 \%)$ & $25.8(8.7 \%)$ \\
\hline Burn & $321.2(100 \%)$ & $70.3(21.9 \%)$ & $64.3(20.0 \%)$ & $91.0(28.3 \%)$ & $40.2(12.5 \%)$ & $13.7(4.3 \%)$ & $9.3(2.9 \%)$ & $32.4(10.1 \%)$ \\
\hline Sharp object & $229.8(100 \%)$ & $70.4(30.6 \%)$ & $57.9(25.2 \%)$ & $48.8(21.2 \%)$ & $14.6(6.4 \%)$ & $10.6(4.6 \%)$ & $8.0(3.5 \%)$ & $19.5(8.5 \%)$ \\
\hline Blunt object & $224.1(100 \%)$ & $63.4(28.3 \%)$ & 49.5 (22.1\%) & $54.6(24.4 \%)$ & $13.7(6.1 \%)$ & $8.8(3.9 \%)$ & $10.6(4.8 \%)$ & $23.4(10.4 \%)$ \\
\hline Assault & $166.9(100 \%)$ & $44.7(26.8 \%)$ & $38.8(23.2 \%)$ & $35.7(21.4 \%)$ & $14.1(8.5 \%)$ & $7.0(4.2 \%)$ & $8.3(5.0 \%)$ & $18.3(10.9 \%)$ \\
\hline Others & $240.4(100 \%)$ & $57.3(23.8 \%)$ & $48.1(20.0 \%)$ & $54.3(22.6 \%)$ & $15.5(6.4 \%)$ & 7.7 (3.2\%) & $19.8(8.3 \%)$ & $22.3(9.3 \%)$ \\
\hline \multicolumn{9}{|l|}{ MAIS } \\
\hline 1 & $121.9(100 \%)$ & $27.2(22.3 \%)$ & $29.4(24.1 \%)$ & $26.9(22.1 \%)$ & $9.0(7.4 \%)$ & $4.5(3.7 \%)$ & $8.2(6.7 \%)$ & $16.7(13.7 \%)$ \\
\hline 2 & $234.3(100 \%)$ & $51.6(22.0 \%)$ & $57.6(24.6 \%)$ & $59.0(25.2 \%)$ & $19.3(8.3 \%)$ & $12.3(5.3 \%)$ & $10.9(4.7 \%)$ & $22.5(9.6 \%)$ \\
\hline 3 & $302(100 \%)$ & $81.4(26.9 \%)$ & 73.6 (24.4\%) & $65.4(21.7 \%)$ & $23(7.6 \%)$ & $17.1(5.7 \%)$ & $14.6(4.8 \%)$ & $26.9(8.9 \%)$ \\
\hline 4 & $379.2(100 \%)$ & $90.5(23.9 \%)$ & $79.1(20.9 \%)$ & $113.1(29.8 \%)$ & $31.2(8.2 \%)$ & $13.3(3.5 \%)$ & $23.7(6.3 \%)$ & $28.1(7.4 \%)$ \\
\hline 5 & $485.3(100 \%)$ & $137.1(28.2 \%)$ & $105.2(21.7 \%)$ & $71.0(14.6 \%)$ & 39.0 (8.0\%) & $16.3(3.4 \%)$ & $59.1(12.2 \%)$ & $23.2(4.8 \%)$ \\
\hline \multicolumn{9}{|c|}{ Principal injured body region } \\
\hline Head & $287.3(100 \%)$ & $63.0(21.9 \%)$ & $72.0(25.1 \%)$ & $67.0(23.3 \%)$ & $21.9(7.6 \%)$ & $16.8(5.8 \%)$ & $19.2(6.7 \%)$ & 23.8 (8.3\%) \\
\hline Face & $148.2(100 \%)$ & $29.6(20.0 \%)$ & $39.4(26.6 \%)$ & $25.3(17.1 \%)$ & $10.2(6.9 \%)$ & $15.3(10.3 \%)$ & $7.2(4.9 \%)$ & $21.1(14.2 \%)$ \\
\hline Spine & $205.1(100 \%)$ & 46.9 (22.9\%) & 55.1 (26.9\%) & 39.7 (19.3\%) & $16.6(8.1 \%)$ & $9.4(4.6 \%)$ & $18.6(9 \%)$ & $18.8(9.2 \%)$ \\
\hline Thorax/abdomen & $208.7(100 \%)$ & $46.6(22.3 \%)$ & $50.0(24.0 \%)$ & $55.8(26.7 \%)$ & $13.8(6.6 \%)$ & $8.1(3.9 \%)$ & $13.8(6.6 \%)$ & $20.5(9.8 \%)$ \\
\hline Upper extremity & $238.4(100 \%)$ & $65.1(27.3 \%)$ & $55.3(23.2 \%)$ & $57.5(24.1 \%)$ & $18.5(7.8 \%)$ & $10.8(4.5 \%)$ & $9.0(3.8 \%)$ & $22.2(9.3 \%)$ \\
\hline Lower extremity & $335.8(100 \%)$ & $93.8(27.9 \%)$ & $75.2(22.4 \%)$ & $80.1(23.9 \%)$ & $28.8(8.6 \%)$ & $13.8(4.1 \%)$ & $14.1(4.2 \%)$ & $29.1(8.7 \%)$ \\
\hline \multicolumn{9}{|l|}{ Surgery } \\
\hline Non-surgery & $194.2(100 \%)$ & $0.0(0 \%)$ & $58.8(30.3 \%)$ & $76.1(39.2 \%)$ & $18.6(9.6 \%)$ & $6.2(3.2 \%)$ & $18.2(9.4 \%)$ & $16.4(8.4 \%)$ \\
\hline Minor surgery & $201.9(100 \%)$ & $67.8(33.6 \%)$ & 49.2 (24.4\%) & 25.2 (12.5\%) & $14.9(7.4 \%)$ & 9.4 (4.6\%) & $10(5 \%)$ & 22 (10.9\%) \\
\hline Major surgery & $427.8(100 \%)$ & $128.2(30 \%)$ & $89.8(21 \%)$ & $99.3(23.2 \%)$ & $32.2(7.5 \%)$ & $26.5(6.2 \%)$ & $16.7(3.9 \%)$ & $34.5(8.1 \%)$ \\
\hline \multicolumn{9}{|l|}{ Admission route } \\
\hline Direct & $251.2(100 \%)$ & $63.6(25.3 \%)$ & $61.3(24.4 \%)$ & $56.9(22.7 \%)$ & $19.0(7.6 \%)$ & $15.8(6.3 \%)$ & $11.2(4.4 \%)$ & $23.2(9.3 \%)$ \\
\hline Indirect-lower & $278.4(100 \%)$ & $69.3(24.9 \%)$ & $66.1(23.7 \%)$ & $68.5(24.6 \%)$ & $23.6(8.5 \%)$ & $10.9(3.9 \%)$ & $14.9(5.4 \%)$ & $24.1(8.7 \%)$ \\
\hline Indirect-higher & $380.4(100 \%)$ & $82.4(21.7 \%)$ & $84.8(22.3 \%)$ & $83.8(22.0 \%)$ & $28.2(7.4 \%)$ & $13.9(3.6 \%)$ & $38.3(10.1 \%)$ & $33.5(8.8 \%)$ \\
\hline
\end{tabular}

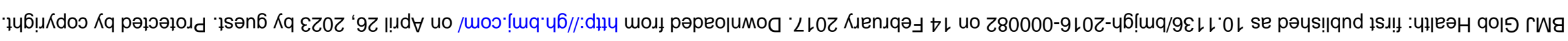


Table 5 Role of insurance and medical care costs during hospitalisation (generalised linear models, unadjusted and adjusted by demographic and injury characteristics)

\begin{tabular}{|c|c|c|c|c|c|c|c|c|}
\hline & \multicolumn{8}{|l|}{ Cost categories } \\
\hline & \multicolumn{2}{|c|}{ Total medical costs } & \multicolumn{2}{|l|}{ Surgery cost } & \multicolumn{2}{|c|}{ Test/examination cost } & \multicolumn{2}{|l|}{ Drug cost } \\
\hline & Unadjusted $^{\star}$ & Adjusted $^{\star \star}$ & Unadjusted $^{*}$ & Adjusted $^{\star \star}$ & Unadjusted $^{\star}$ & Adjusted $^{\star \star}$ & Unadjusted $^{\star}$ & Adjusted $^{\star \star}$ \\
\hline \multicolumn{9}{|l|}{ Insurance } \\
\hline No & 1.00 & 1.00 & 1.00 & 1.00 & 1.00 & 1.00 & 1.00 & 1.00 \\
\hline Yes & $0.88(0.056)$ & $0.93(0.142)$ & $0.82(0.092)$ & $0.88(0.291)$ & $0.93(0.258)$ & $0.97(0.675)$ & $0.88(0.201)$ & $0.92(0.314)$ \\
\hline \multicolumn{9}{|l|}{ Gender } \\
\hline Male & & 1.00 & & 1.00 & & 1.00 & & 1.00 \\
\hline Female & & $1.07(0.108)$ & & $1.11(0.379)$ & & $1.10(0.11)$ & & $0.99(0.885)$ \\
\hline \multicolumn{9}{|l|}{ Admission route } \\
\hline Direct & & 1.00 & & 1.00 & & 1.00 & & 1.00 \\
\hline $\begin{array}{l}\text { Indirect- } \\
\text { lower }\end{array}$ & & $1.01(0.796)$ & & $0.98(0.844)$ & & $0.99(0.894)$ & & $1.08(0.294)$ \\
\hline $\begin{array}{l}\text { Indirect- } \\
\text { higher }\end{array}$ & & $1.06(0.503)$ & & $0.77(0.219)$ & & $0.97(0.807)$ & & $1.07(0.659)$ \\
\hline \multicolumn{9}{|l|}{ External cause } \\
\hline All others & & 1.00 & & 1.00 & & 1.00 & & 1.00 \\
\hline Falls & & $0.88(0.067)$ & & $0.65(0.018)$ & & $1.10(0.253)$ & & $0.89(0.344)$ \\
\hline RTIs & & $0.90(0.080)$ & & $0.78(0.124)$ & & $1.04(0.598)$ & & $0.81(0.04)$ \\
\hline \multicolumn{9}{|l|}{ MAIS } \\
\hline 1 & & 1.00 & & 1.00 & & 1.00 & & 1.00 \\
\hline 2 & & $1.49(<0.01)$ & & $2.03(0.001)$ & & $1.57(<0.01)$ & & $1.39(0.017)$ \\
\hline 3 & & $1.59(<0.01)$ & & $2.53(<0.01)$ & & $1.72(<0.01)$ & & $1.21(0.171)$ \\
\hline 4 & & $1.74(<0.01)$ & & $1.99(0.016)$ & & $1.82(<0.01)$ & & $1.75(0.002)$ \\
\hline 5 & & $2.65(<0.01)$ & & $4.07(<0.01)$ & & $2.60(<0.01)$ & & $1.67(0.034)$ \\
\hline \multicolumn{9}{|c|}{ Principal injured region } \\
\hline Face & & 1.00 & & 1.00 & & 1.00 & & 1.00 \\
\hline $\begin{array}{l}\text { Spine/thorax/ } \\
\text { abdomen }\end{array}$ & & $1.24(0.015)$ & & $1.19(0.466)$ & & $1.07(0.538)$ & & $1.52(0.007)$ \\
\hline $\begin{array}{l}\text { Upper } \\
\text { extremity }\end{array}$ & & $1.06(0.474)$ & & $1.10(0.668)$ & & $0.98(0.837)$ & & $1.38(0.039)$ \\
\hline $\begin{array}{l}\text { Hip/lower } \\
\text { extremity }\end{array}$ & & $1.23(0.009)$ & & $1.33(0.198)$ & & $1.14(0.198)$ & & $1.4(0.019)$ \\
\hline Head & & $1.43(<0.01)$ & & $1.21(0.367)$ & & $1.32(0.005)$ & & $2.19(<0.01)$ \\
\hline \multicolumn{9}{|l|}{ Surgery } \\
\hline No & & 1.00 & & 1.00 & & 1.00 & & 1.00 \\
\hline Yes & & $1.62(<0.01)$ & & $2.29(<0.01)$ & & $1.33(<0.01)$ & & $1.85(<0.01)$ \\
\hline Length of stay & & $1.09(<0.01)$ & & $1.11(<0.01)$ & & $1.07(<0.01)$ & & $1.11(<0.01)$ \\
\hline $\begin{array}{l}\text { Residual } \\
\text { deviance } \\
\text { (goodness-of-fit } \\
\text { measure) }\end{array}$ & 634.16 & 258.07 & 755.92 & 550.68 & 618.73 & 402.52 & 1033.20 & 646.75 \\
\hline
\end{tabular}

${ }^{*}$ GLM model with cost as the dependent variable and insurance status as the only independent variable.

${ }^{\star *}$ GLM model with cost as the dependent variable and insurance status as the independent variable controlling for other demographic (age, gender and occupation) and injury characteristics (external cause, severity, principal body region injured, hospital transfer status and surgery status).

during hospitalisation were surgery, diagnostic tests/ examinations and drugs. These were similar to results in a study of determinants of costs of RTIs in an Indian city. ${ }^{14}$ Their major cost drivers were also surgery, tests/ examinations and drugs. However, the proportions represented by surgery, tests/examinations and drugs were $34 \%, 24 \%$ and $34 \%$, respectively. ${ }^{14}$ While surgery cost is still the largest proportion in the current study, it seems to vary across settings. In another study on costs of RTIs, falls and burns in India, the proportion of cost for surgery in the total medical care costs was $26 \%{ }^{15}$

In addition to the significant contribution of surgery to the total costs, we found that a large proportion of these were paid for diagnostic test/examination and drugs. In combination, these two categories contributed nearly $50 \%$ of the total. This can be related to an issue commonly found in low-income countries (LMIC), supply of unnecessary care induced by providers. This is 
common in LMICs because these are where professional regulation and supervision are poorly enforced, the public health sector is underfunded and the providers are mainly paid through a retrospective provider payment mechanism, such as a fee-for-service reimbursement mechanism. ${ }^{16}$ In Vietnam, the rolling-out of financial autonomy in government hospital policy since early 2000s provides hospital opportunities to generate additional resources. The policy has helped hospitals improving their financial sustainability and able to provide wider range of healthcare services. ${ }^{17}$ However, to increase revenue, providers tend to overprescribe diagnosis tests, examinations and medications, including costly brand-name drugs. ${ }^{17} 18 \mathrm{~A}$ study of diagnostic testing provides a large variation in the rates of testing cross hospitals in a province, ranging from 0.3 tests per patient visit to 6.4. Among 200 patients who had a CT scan, $80 \%$ had an ultrasound. Such level of care was considered excessive and could be related to financial incentives or other non-medical reasons. ${ }^{19}$ In terms of drug use, Nguyen $e t a l^{20}$ found that in 2005, medicine normally accounted for over $50 \%$ of total household total healthcare expenditure. In addition, low-price generic drugs were generally less available in public sector facilities than brand-name drugs and patients usually paid 46.6 and 11.4 times the international reference prices for brand-name and generic drugs, respectively. ${ }^{20}{ }^{21}$ Although we were unable to explore this in more detail, it is a plausible mechanism for the high costs for diagnostic test/examination and drugs in this study.

Hospitalisation creates a significant financial burden to the family. Earlier work from the same cohort shows that as many as $27 \%$ of families faced catastrophic expenditure and the risk was even higher for those without health insurance. ${ }^{5}$ However, analyses of the effect of health insurance on the three major costs drivers in the current analysis indicate that there was no statistically significant difference for each of the cost categories between patients with and without health insurance. Health insurance was introduced at around the same time as the hospital user fee in early 1990s. It is meant to be a mechanism to protect patient from financial burden resulted from the introduction of the user fee. However, after nearly 20 years of implementation and continuous amendments, a number of studies have indicated the modest benefits of health insurance in helping patients away from catastrophe of medical expense. ${ }^{22-24}$ In fact, out-of-pocket payment was too high $^{23}$ and no statistically significant effect of health insurance on the level of out-of-pocket payment was found. In addition, payments, Nguyen ${ }^{22}$ did not find any.

In another study using data from five Vietnam Living Standard Surveys from 2002 to 2010, Hoang et al revealed that the financial protection functionality of the health insurance in Vietnam was limited and not consistent over time. The statistically significantly lower rates of catastrophic expenditure among household with health insurance were found only in 2004 and $2006 .{ }^{24}$ The current study provides another evidence of the uncertainty, specifically for injury treatment, in the capacity of health insurance in protecting patients from high costs.

Our study also shows a low level of health insurance usage. While the Ministry of Health claimed that the national coverage of health insurance was $61 \%$ by $2010,{ }^{8}$ in this study, only $25 \%$ of study participants reported to have their insurance. The cumbersome procedures to access insurance benefits are often reported as the reason why patients did not use their insurance. Rather than risking a longer wait time and lower quality of care, some insured patients may just forgo all insurance benefits and incur full out-of-pocket payment. ${ }^{25}$ From the provider perspective, a disincentive to treat insured patients exists because of the inappropriate fee structure and the way providers are reimbursed. Partial payments from users and health insurance reimbursement are not always sufficient to cover the costs of providing healthcare services. ${ }^{7}$ Such differential treatment between insured patients, particularly those with low premiums, and private fee-paying patients would strongly influence their decision to use or not to use their insurance.

While revealing important findings on medical care cost categories and role of health insurance for injury treatment, it is also important to note some study limitations. First, our study reported only participants' out-of-pocket payments during their hospitalisation. These were not the actual costs paid to the hospital because the amount that hospital would be reimbursed by insurance was not included. This is because our focus was to measure the financial burden to the patients and their families. The actual costs would be higher than our estimates if all costs were included. Second, the analyses for specific schemes of health insurance were not conducted because participants' exact health insurance scheme was not available. While many schemes exist, they can be grouped in four major categories, including the compulsory health insurance (covering formal-sector employees, retirees, the disabled, veterans and their dependents), voluntary health insurance (opening to not eligible for coverage under compulsory health insurance), health insurance for the poor and free health insurance for children under $6 .^{7}$ Their level of reimbursement or out-of-pocket payment would vary depending on the scheme they were on. The lack of this information, a kind of endogeneity bias, would potentially understate, especially when the insurance reimbursement was significant, the effect of insurance status on out-of-pocket payments.

\section{CONCLUSION}

This study is one of very few studies reporting estimates of specific categories of medical care costs paid out-of-pocket by injury patients in a low-income setting. The high total costs explicitly highlight the need of primary prevention, not only to protect patients from the financial burden but also losses in health and quality of life. The analyses on the effect of health 
insurance on total and specific category costs provide further evidence of the current problems in the implementation of health insurance in Vietnam. There is an urgent need of comprehensive reform in the system, to avoid unnecessary services and receive best quality of care at affordable costs for patients.

\section{Handling editor Sanni Yaya.}

Contributors $\mathrm{HN}, \mathrm{RI}$, SJ and CP conceived the study, developed and refined the study design, instrument and analysis plan. HN analysed the data and wrote the draft. RI provided feedback regarding analyses and provided critical revision of draft manuscripts.

Funding This study was funded by Atlantic Philanthropies (grant number AP15333). The funder had no direct involvement with the scientific aspects of the study.

\section{Competing interests None declared.}

Ethics approval The Human Ethics Committee in University of Sydney, Australia, and the Hanoi School of Public Health Ethics Committee and Thai Binh General Hospital in Vietnam.

Provenance and peer review Not commissioned; externally peer reviewed.

Data sharing statement No additional data are available.

Open Access This is an Open Access article distributed in accordance with the Creative Commons Attribution Non Commercial (CC BY-NC 4.0) license, which permits others to distribute, remix, adapt, build upon this work noncommercially, and license their derivative works on different terms, provided the original work is properly cited and the use is non-commercial. See: http:// creativecommons.org/licenses/by-nc/4.0/

\section{REFERENCES}

1. World Health Organization. Violence and injuries: the facts 2014 Geneva: World Health Organization, 2015.

2. World Health Organization. Global Health Estimates Summary Tables: 20 Leading Causes of Death. 2013. 2013 (08 Mar 2016); http://www.who.int/healthinfo/global_burden_disease/projections/en/

3. Administration of Preventive Medicine. Ministry of Health, National mortality statistics in 2010. Hanoi: Ministry of Health, 2012.

4. Center for Injury Policy and Prevention Research. Vietnam National Injury Survey 2010. Hanoi: Hanoi School of Public Health, 2012.

5. Nguyen $\mathrm{H}$, Ivers $\mathrm{R}$, Jan $\mathrm{S}$, et al. Catastrophic household costs due to injury in Vietnam. Injury 2013;5:684-90.

6. Xu K, Evans DB, Kawabata K, et al. Household catastrophic health expenditure: a multicountry analysis. Lancet 2003;9378:111-17.

7. Ministry of Health and Health Partnership Group. Joint Annual Health Review 2008-health financing in Vietnam. Hanoi: Vietnam, 2009.
8. Ministry of Health and Health Partnership Group. Joint Annual Health Review 2010-Vietnam's health system on the threshold of the five-year plan 2011-2015. Hanoi: Vietnam, 2011.

9. Thai Binh Department of Health. Report on statistics of injuries in 12-month 2010. Thai Binh: Thai Binh Department of Health, 2011.

10. Nguyen H. Injury, a significant cause of poverty: evidence from a prospective cohort study of adult injuries in Thai Binh province, Vietnam, in School of Public Health. Sydney: University of Sydney, 2012:266.

11. Manning WG, Mullahy J. Estimating log models: to transform or not to transform? J Health Econ 2001;4:461-94.

12. Stata Web Books. Regression with Stata-Chapter 2-Regression Diagnostics. UCLA: Academic Technology Services, Statistical Consulting Group. 15 Aug 2011. http://www.ats.ucla.edu/stat/stata/ webbooks/reg/chapter2/statareg2.htm

13. Johnson PE. GLM \#2: Residuals and analysis of fit. Stat writeups. 2016. (cited 25 Oct 2016).

14. Reddy GM, Negandhi H, Singh D, et al. Extent and determinants of cost of road traffic injuries in an Indian city. Indian J Med Sci 2009;12:549-56.

15. Reddy GM, Singh D, Singh A. Community based estimation of extent and determinants of cost of injuries in a North Indian city. Indian J Med Sci 2012;12:23-9.

16. Sepehri A. Does autonomization of public hospitals and exposure to market pressure complement or debilitate social health insurance systems? Evidence from a low-income country. Int $J$ Health Serv 2014;1:73-92.

17. Ministry of Health. Health Strategy and Policy Institute, and Bank W, Lessons for Hospital Autonomy Implementation in Vietnam from International Experience. Hanoi: World Bank, 2011.

18. Vian T, Brinkerhoff DW, Feeley FG, et al. Confronting corruption in the health sector in Vietnam: patterns and prospects. Public Adm Dev 2012;1:49-63.

19. Vyan T. Corruption perceptions and impact on poverty in the health sector in Vietnam: how to improve transparency and accountability: report of the donors roundtable. Hanoi: Towards Transparency and Embassy of Sweden, 2010.

20. Nguyen A, Knight R, Mant A, et al. Medicine pricing policies: lessons from Vietnam. South Med Rev 2010;3:12-19.

21. Nguyen A, Knight R, Mant A, et al. Medicine prices, availability, and affordability in Vietnam. South Med Rev 2009;2:2-9.

22. Nguyen CV. The impact of voluntary health insurance on health care utilization and out-of-pocket payments: new evidence for Vietnam. Health Econ 2012;21:946-66.

23. Nguyen KT, Khuat OT, Ma S, et al. Impact of health insurance on health care treatment and cost in Vietnam: a health capability approach to financial protection. Am J Public Health 2012;8:1450-61.

24. Van Minh H, Kim Phuong NT, Saksena P, et al. Financial burden of household out-of pocket health expenditure in Viet Nam: findings from the National Living Standard Survey 2002-2010. Soc Sci Med 2013;96:258-63.

25. Sepehri A, Sarma S, Serieux J. Who is giving up the free lunch? The insured patients' decision to access health insurance benefits and its determinants: evidence from a low-income country. Health Policy 2009;92(2-3):250-8. 